\title{
Blood platelet kinetics and platelet transfusion
}

\author{
Richard H. Aster \\ BloodCenter of Wisconsin, Medical College of Wisconsin, Milwaukee, Wisconsin, USA.
}

\begin{abstract}
The discovery of citrate anticoagulant in the 1920s and the development of plastic packs for blood collection in the 1960s laid the groundwork for platelet transfusion therapy on a scale not previously possible. A major limitation, however, was the finding that platelet concentrates prepared from blood anticoagulated with citrate were unsuitable for transfusion because of platelet clumping. We found that this could be prevented by simply reducing the $\mathrm{pH}$ of platelet-rich plasma to about 6.5 prior to centrifugation. We used this approach to characterize platelet kinetics and sites of platelet sequestration in normal and pathologic states and to define the influence of variables such as anticoagulant and $\mathrm{ABO}$ incompatibility on post-transfusion platelet recovery. The "acidification" approach enabled much wider use of platelet transfusion therapy until alternative means of producing concentrates suitable for transfusion became available.
\end{abstract}

The identification of platelets as a distinct cellular element of blood with a critical role in hemostasis in the late 1800s (1) inevitably led to speculation about platelet transfusion as a treatment for bleeding in patients with thrombocytopenia. The realization of this goal was delayed for many years by technical barriers. Development of citrate-based anticoagulants in the 1920 s and flexible plastic blood containers in the 1950s-1960s made it feasible to collect blood in a plastic pack containing standard acid-citrate-dextrose (ACD) anticoagulant, centrifuge it slowly, and express the supernatant platelet-rich plasma (PRP) into a plastic side-pack for convenient transfusion. Early studies showed that platelets from multiple units of blood were needed to achieve a therapeutic effect in a bleeding patient. To prevent volume overload, this required that platelets be concentrated before being transfused. The obvious way to accomplish this was to centrifuge PRP at high speed, remove the supernatant plasma, and suspend the pelleted platelets in a small volume by gently massaging the plastic pack. It soon became apparent that concentrates prepared in this way almost invariably contained large and small platelet aggregates and few single platelets. Not surprisingly, clinicians were reluctant to transfuse these preparations.

It was known at this time that platelets isolated from blood that had been antico-

Conflict of interest: The author has declared that no conflict of interest exists.

Citation for this article: J Clin Invest. 2013; 123(11):4564-4565. doi:10.1172/JCI70335. agulated with EDTA could be pelleted from PRP by centrifugation and dispersed without difficulty. To meet the growing demand for platelet transfusions, the Fenwal Company developed the "EDTA Platelet Pack," consisting of a plastic collection bag containing EDTA and an attached satellite bag into which PRP could be expressed, concentrated by centrifugation into a pellet, and suspended in a small volume of plasma. Red cells were returned to the donor to enable repeated platelet donations. Despite the obvious limitations of this approach, thousands of pooled EDTA platelet concentrates were transfused in the late 1950s and the 1960s. This procedure was labor intensive, and its application was restricted to relatively few, critically ill patients.

In 1961, Gardner and associates conducted seminal studies to define the pathophysiology of various thrombocytopenic disorders $(2,3)$. They labeled EDTA platelets with $\mathrm{NaCr}^{51} \mathrm{O}_{4}$ to follow the cells after transfusion. In these studies, very few labeled platelets were detected in the peripheral blood during the first few hours after transfusion. After this time, a variable number of cells reentered circulation. The immediate sequestration of a large fraction of the transfused cells, possibly in the liver and lung (4), followed by the eventual return of some platelets into circulation was considered to be a consequence of the labeling procedure.

At this time, working at the Thorndike Memorial Laboratory of the Boston City Hospital, we were similarly interested in studying platelet kinetics, and we confirmed the findings of Gardner and coworkers about the circulation kinetics of EDTA platelets. We examined PRP prepared from EDTA and ACD whole blood under phase microscopy and noted that platelet morphology was quite different in the two preparations. In ACD preparations, platelets were discoid in shape, but in EDTA preparations, they assumed an irregular, almost spherical configuration. Another striking difference was the appearance of PRP examined in a light beam while being gently agitated: ACD platelets shimmered and swirled, whereas an EDTA platelet suspension was uniform in appearance throughout. We wondered whether structural changes induced in platelets by EDTA explained the failure of most of these platelets to circulate after transfusion and carried out studies to determine whether platelet clumping in concentrates from ACD-prepared PRP could be prevented. Evaluation of several variables revealed that when the $\mathrm{pH}$ of ACD PRP was reduced from its starting value of about 7.2 to about 6.5 before centrifugation, the pelleted platelets could readily be dispersed, yet retained their normal discoid shape. In whole blood or in PRP, this degree of "acidification" could be achieved by simply adding an extra quantity of ACD, the anticoagulant then used routinely for blood collection. The apparent benefit of acidification persisted through repeated centrifugations and made it possible to characterize recovery and survival of ACD platelets in normal subjects (5). Our studies revealed that about $75 \%$ of the labeled ACD platelets were recovered in the recipient immediately after transfusion (Figure 1). After the initial transfusion, the presence of labeled platelets in the blood steadily declined over nine days. In contrast, labeled EDTA platelets peaked in the blood around one day after transfusion and steadily declined afterward (Figure 1). Scanning of body organs with a directional scintillation counter revealed that most of the radioactivity from ACD platelets not recovered in the blood was initially present in the spleen; however, transfused EDTA platelets mainly concentrated in the liver. As ACD platelets were cleared from the circulation, $\mathrm{Cr}^{51}$ accumulated in the liver and spleen, indicating that these organs are the 


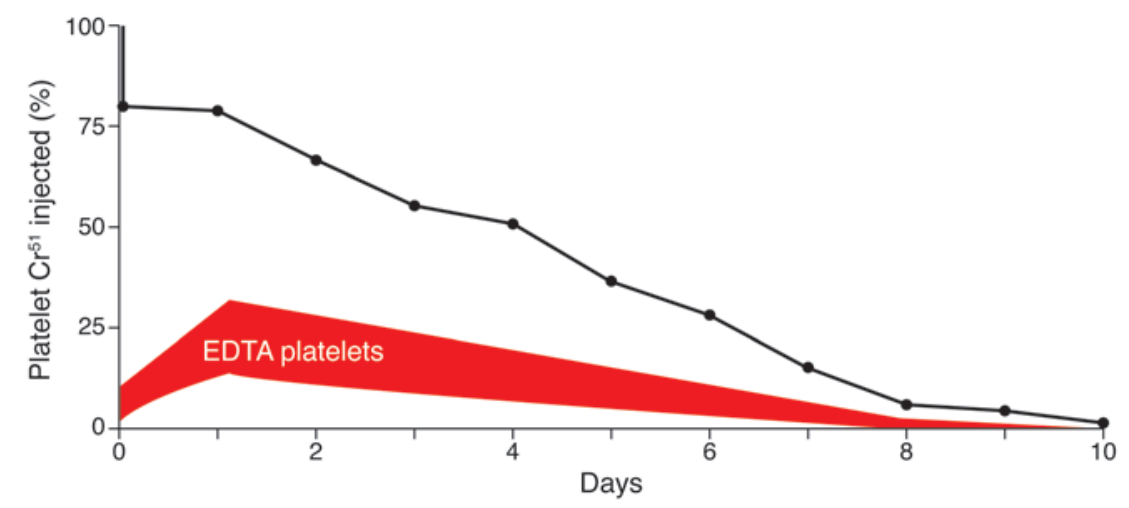

Figure 1

Survival of autologous "citrate platelets" after transfusion to a normal subject. Approximately $75 \%$ of labeled platelets were recovered in the circulation immediately after being transfused. The red area denotes the range of blood platelet radioactivity after the injection of $\mathrm{Cr}^{51}$-labeled "EDTA platelets" on 10 occasions in 7 normal subjects. Adapted from ref. 5.

major sites of platelet deposition. The linear clearance pattern suggested that under normal circumstances, platelets die as a consequence of "senescence," rather than being randomly utilized (5).

Freireich and his colleagues at the National Cancer Institute soon confirmed the superiority of platelet concentrates prepared from acidified ACD blood in producing sustained platelet increases in thrombocytopenic patients (6). Over the next few years, this simple maneuver facilitated much wider use of platelet transfusions, especially in patients being treated for hematologic malignancies. We used the new methodology to characterize platelet clearance and sites of sequestration in normal individuals $(5,7)$ and in patients with platelet destruction mediated by alloantibodies (8) and autoantibodies (9), as well as to more fully define the role of anticoagulants and ABO incompatibility on recovery and survival of transfused platelets (10). We also demonstrated that "hypersplenic" thrombocytopenia is largely caused by pooling of a significant fraction of the total circulating platelet mass in an enlarged spleen, rather than being a consequence of suppressed platelet production or premature platelet destruction (11).
Although acidifying citrated blood or PRP to prepare platelet concentrates for transfusion represented a significant improvement over what was previously possible, other advances soon followed. Mourad found that platelet concentrates prepared from nonacidified ACD blood could be manually suspended with little clumping, provided the platelet pellet was allowed to rest for some time at room temperature before manipulation (12). Other key developments were the finding by Murphy et al. that platelet viability is best maintained by storage at room temperature (13) and the evolution of pheresis systems for isolating large quantities of platelets from single donors.

To my knowledge, reversible aggregation of platelets pelleted from citrated PRP is still not fully understood, but it seems almost certain that fibrinogen binding to partially activated $\alpha_{\mathrm{IIb}} \beta_{3}$ integrin (GPIIb/IIIa) is involved, since fibrinogendependent platelet aggregation is markedly inhibited at pH $6.5(6,14)$. The EDTAinduced structural changes in platelets were well characterized by White (15). The "swirling" of platelets was shown to be a consequence of their normal discoid shape and to correlate fairly well with post-transfusion viability (16).

\section{Acknowledgments}

Supported in part by grant HL-13629 from the National Heart, Lung, and Blood Institute.

Address correspondence to: Richard H. Aster, BloodCenter of Wisconsin, Medical College of Wisconsin, P.O. Box 2178, Milwaukee, Wisconsin 53201, USA. Phone: 414.937.6338; Fax: 414.937.6284; E-mail: richard.aster@bcw.edu.

1. Bizzozero G. Uber einen neuen formbestandteil des blutes und dessen rolle bei der thrombose und blutgerinnung. Virchows Arch Pathol Anat Physiol Klin Med. 1882;90:261-332.

2. Cohen P, Gardner FH, Barnett GO. Reclassification of the thrombocytopenias by the Cr51-labeling method for measuring platelet life span. $N$ Engl J Med. 1961;264:1294-1299.

3. Cohen P, Gardner FH, Barnett GO. Reclassification of the thrombocytopenias by the Cr51-labeling method for measuring platelet life span. $N$ EnglJ Med. 1961;264:1350-1355.

4. Aas KA, Gardner FH. Survival of blood platelets labeled with chromium. J Clin Invest. 1958; 37(9):1257-1268.

5. Aster RH, Jandl JH. Platelet sequestration in man. I. Methods. J Clin Invest. 1964;43:843-855.

6. Flatow FA, Freireich EJ. The increased effectiveness of platelet concentrates prepared in acidified plasma. Blood. 1966;27(4):449-459.

7. Aster RH. Studies of the fate of platelets in rats and man. Blood. 1969;34(2):117-128.

8. Aster RH, Jandl JH. Platelet sequestration in man. II. Immunological and clinical studies. J Clin Invest. 1964;43:856-869.

9. Aster RH, Keene WR. Sites of platelet destruction in idiopathic thrombocytopenic purpura. BrJ Haematol. 1969;16(1):61-73.

10. Aster RH. Effect of anticoagulant and ABO incompatibility on recovery of transfused human platelets. Blood. 1965;26(6):732-743.

11. Aster RH. Pooling of platelets in the spleen: role in the pathogenesis of "hypersplenic" thrombocytopenia. J Clin Invest. 1966;45(5):645-657.

12. Mourad N. A simple method for obtaining platelet concentrates free of aggregates. Transfusion. 1968; 8(1):48.

13. Murphy S, Gardner FH. Effect of storage temperature on maintenance of platelet viability - deleterious effect of refrigerated storage. NEngl J Med. 1969; 280(20):1094-1098.

14. Peerschke EI, Zucker MB, Grant RA, Egan JJ, Johnson MM. Correlation between fibrinogen binding to human platelets and platelet aggregability. Blood. 1980;55(5):841-847.

15. White JG. Effects of ethylenediamine tetracetic acid (EDTA) on platelet structure. Scand J Haematol. 1968; 5(4):241-254.

16. Brecher ME, Hay SN. Transfusion medicine illustrated. Platelet swirling. Transfusion. 2004;44(5):627. 